"(c) 2017 IEEE. Personal use of this material is permitted. Permission from IEEE must be obtained for all other users, including reprinting/ republishing this material for advertising or promotional purposes, creating new collective works for resale or redistribution to servers or lists, or reuse of any copyrighted components of this work in other works. 


\title{
Truncated Circular Cone, Slot Antenna Array that Radiates a Circular Polarized Conical Beam
}

\author{
Guanshen Chenhu, Junping Geng, Senior Member, IEEE, Han Zhou, Jianping Li, Liang liu, Xianling Liang, Senior \\ Member, IEEE, Weiren Zhu, Member, IEEE, Ronghong Jin, Fellow, IEEE, Richard W. Ziolkowski, Fellow, IEEE
}

\begin{abstract}
A truncated circular cone slot array antenna that radiates a circular polarized $(C P)$ conical beam is presented. The direction into which the conical beam radiates depends mainly on the tilt angle of the outer conductor as well as the proper arrangement of the positions of its two slot arrays. The $\mathrm{CP}$ property is realized by introducing quasi-perpendicular slots that are cut into the outer conductor of a truncated circular cone. To illustrate the performance characteristics of this $\mathrm{CP}$ conical beam antenna, the tilt angle is set to be $45^{\circ}$ and the beam angle is pointed at $45^{\circ}$ from boresight within the operating band. A prototype was fabricated and tested. The measured results demonstrate that its -10-dB impedance bandwidth $\left(\left|\mathbf{S}_{11}\right|<-\mathbf{- 1 0} \mathbf{~ d B}\right)$ is $720 \mathrm{MHz}$, from 5.05 to $5.77 \mathrm{GHz}$; and its $3 \mathrm{~dB}$ axial-ratio (AR) bandwidth is $500 \mathrm{MHz}$, from 5.5 to $6.0 \mathrm{GHz}$. Its realized gain over this AR bandwidth ranges from 4.84 to $5.8 \mathrm{~dB}$. The designed beam direction can be easily changed to meet specific application requirements. Moreover, it can be readily reconfigured to be a conformal antenna mounted on the top of a high speed mobile platform.
\end{abstract}

Index Terms-Circular polarization, conformal antenna, conical beam, slot arrays.

\section{INTRODUCTION}

$\mathrm{T}$ HERE has been intense recent interest in antenna systems that radiate a circularly polarized $(\mathrm{CP})$ conical beam. For instance, circular patch antennas with multi-feed probes have been employed to generate CP conical beams [1], [2] by means of a $\mathrm{TM}_{21}$ mode approach [3]. A single-feed circular aperture antenna [4] has achieved a radiating flare angle of $28^{\circ}$ and better azimuthal symmetry by exciting simultaneously both the $\mathrm{TM}_{01}$ and $\mathrm{TE}_{01}$ modes in a circular waveguide. Another effort [5] reported a low profile and wideband patch antenna that merged the $\mathrm{TM}_{01}$ and $\mathrm{TM}_{02}$ modes. Unfortunately, none of these antennas can be redesigned to radiate a large angle beam along the boresight direction.

A radial line slot array (RLSA) antenna [6] attained a null in the boresight direction by arranging slots in the aperture of a circular waveguide and by exciting them with a rotational phase along the circumferential direction. It realized a conical beam

Manuscript received May 25, 2017; revised July 0x, 2017.

This work was supported by the National Natural Science Foundation under Grants 61571289, 61571298, and 61471240.

G. Chenhu, J. Geng, H. Zhou, J. Li, L. Liu, X. Liang, W. Zhu, and R. Jin are with the Department of Electronics Engineering, Shanghai Jiao Tong University, Shanghai 200240, China (e-mail: gengjunp@sjtu.edu.cn).

R. W. Ziolkowski is with the University of Technology Sydney, Global Big Data Technologies Centre, Ultimo NSW 2007, Australia and with The University of Arizona, Department of Electrical and Computer Engineering, Tucson, AZ 85721 USA.

Color versions of one or more of the figures in this letter are available online at http://ieeexplore.ieee.org.

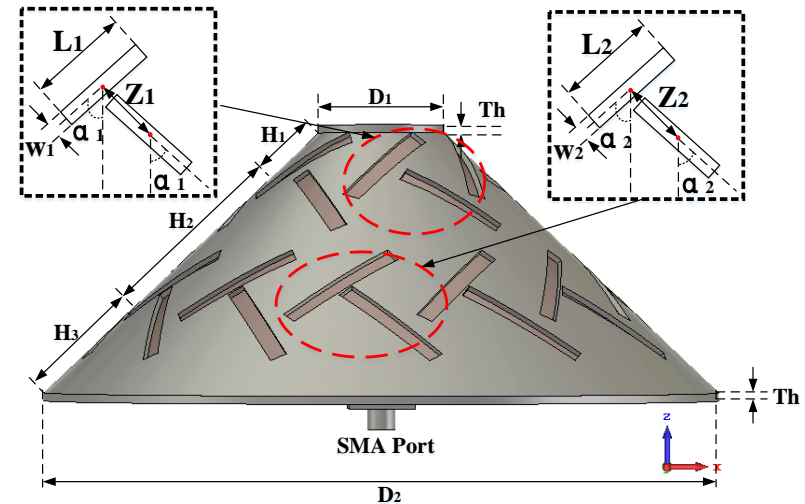

(a)

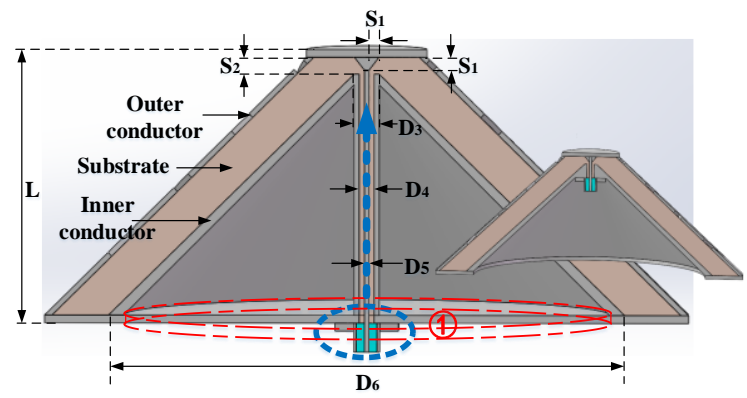

(b)

Fig. 1. Geometry of the CP conical beam antenna. (a) Side view. (b) Cross-sectional view.

with a variable pointing angle by changing the distance between the slot pairs and the waveguide center. Nevertheless, because the aperture is planar, the gain deteriorates as the beam angle gets larger from the boresight. Another approach is to cut four sets of perpendicular slot pairs around the axis of a coaxial cylinder, each set being spaced equally along it, to realize a high gain omnidirectional $\mathrm{CP}$ antenna [7]. It radiates a conical beam at $90^{\circ}$ from boresight. By changing the distance between each set of slot pairs, the beam can be pointed to other angles near to $90^{\circ}$. However, because strong coupling between the slot pairs is critical and the antenna length is restricted, the distance between each set of slot pairs is limited. Consequently, the beam angle coverage is too.

In this paper, taking advantage of the designs in [6] and [7], it is demonstrated that by cutting two sets of quasi-perpendicular slots into the outer conductor of a truncated circular cone, as shown in Fig. 1, a CP conical beam can be produced with no limitation on the angle it points away from boresight. Simulation studies reveal that the direction of the conical beam depends mainly on the slant angle of the outer conductor and the proper arrangement of the positions of these two slot arrays. Because the conical beam can point in any specified direction 


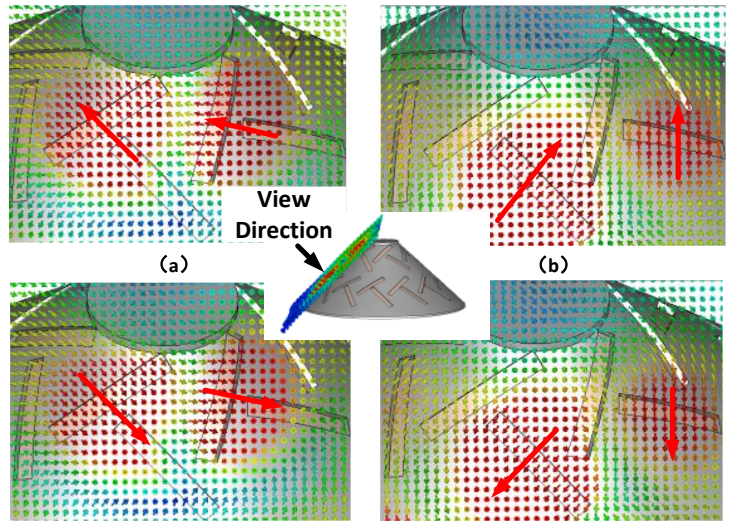

(c)

(d)

Fig. 2. Electric field behavior on the outer conductor of the CP conical beam antenna over one period at the $5.7 \mathrm{GHz}$ source frequency. (a) $\mathrm{t}=0$. (b) $\mathrm{t}=\mathrm{T} / 4$. (c) $\mathrm{t}=2 \mathrm{~T} / 4$. (d) $\mathrm{t}=3 \mathrm{~T} / 4$.

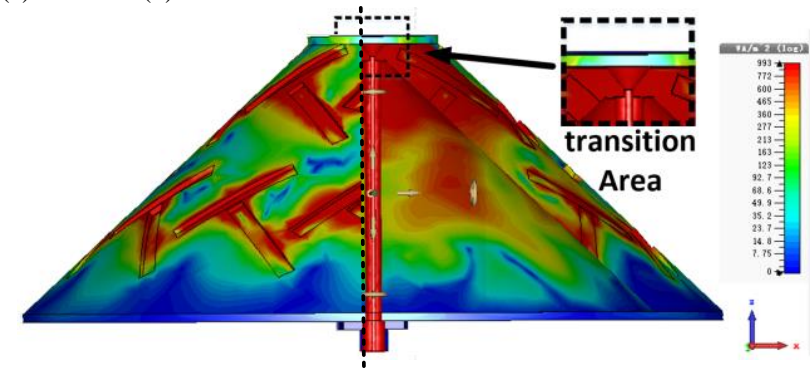

Fig. 3. Power flow on the outer conductor of the CP conical beam antenna at 5.7 GHz. Left: side view, and Right: cross-sectional view.

TABLE I

OPTIMIZED GEOMETRIC PARAMETERS OF THE CP CONICAL BEAM ANTENNA

\begin{tabular}{|c|c|c|c|c|c|}
\hline $\mathrm{D}_{1}$ & $24 \mathrm{~mm}$ & $\mathrm{D}_{2}$ & $128 \mathrm{~mm}$ & $\mathrm{D}_{3}$ & $4.98 \mathrm{~mm}$ \\
\hline $\mathrm{D}_{4}$ & $3 \mathrm{~mm}$ & $\mathrm{D}_{5}$ & $0.92 \mathrm{~mm}$ & $\mathrm{D}_{6}$ & $102 \mathrm{~mm}$ \\
\hline $\mathrm{Th}$ & $1.5 \mathrm{~mm}$ & $\mathrm{H}_{1}$ & $19.8 \mathrm{~mm}$ & $\mathrm{H}_{2}$ & $33.6 \mathrm{~mm}$ \\
\hline $\mathrm{H}_{3}$ & $15.9 \mathrm{~mm}$ & $\mathrm{~S}_{1}$ & $2.4 \mathrm{~mm}$ & $\mathrm{~S}_{2}$ & $3.2 \mathrm{~mm}$ \\
\hline $\mathrm{W}_{1}$ & $3.3 \mathrm{~mm}$ & $\mathrm{~L}_{1}$ & $22 \mathrm{~mm}$ & $\mathrm{~W}_{2}$ & $3.3 \mathrm{~mm}$ \\
\hline $\mathrm{L}_{2}$ & $24.3 \mathrm{~mm}$ & $\mathrm{Z}_{1}$ & $14.3 \mathrm{~mm}$ & $\mathrm{Z}_{2}$ & $14.2 \mathrm{~mm}$ \\
\hline$\alpha_{1}$ & $52.7 \mathrm{deg}$ & $\alpha_{2}$ & $50.9 \mathrm{deg}$ & $\mathrm{L}$ & $52 \mathrm{~mm}$ \\
\hline
\end{tabular}

from boresight, its coverage greatly surpasses those of previously reported systems [6], [7]. A gradient coaxial line is used to achieve impedance matching, as well as to equalize the energy distribution between the two sets of slot pairs and, hence, to realize a high gain. An example antenna with a $45^{\circ}$ slant angle was optimized and fabricated. The tested prototype verifies the design concepts. It has a simple mechanical structure and does not need a complex feed network. Moreover, referring to Fig. 1(b), a large part of the bottom surface conductor (1) can be removed. This enables moving the SMA port upwards and, hence, shortening the inner coaxial cable. This would allow the system to be mounted as a conformal antenna on the top of high speed mobile platform.

\section{ANTENNA DESIGN AND ANALYSIS}

The CP conical beam antenna structure is shown in Fig. 1. The dielectric Teflon $\left(\varepsilon_{\mathrm{r}}=2.1\right)$ was selected as the substrate between the inner and outer conductors of the truncated cone. This gradual tapered transmission line facilitates a relatively wide impedance matching bandwidth. One end of the radiator is shorted to ground and the other is matched to the $50-\Omega$ source. Two sets of quasi-perpendicular slot pairs are cut from the surface of the outer conductor. One of the slots in each pair is inclined at the clockwise angle $\alpha_{i}$ and the other is inclined at the anticlockwise angle $\alpha_{i}(i=1$ for the upper set of slot pairs and $i$ $=2$ for the lower set). Thus, these slots are quasi-perpendicular to each other. The slot pairs serve as two magnetic current elements. The length of each slot $\left(L_{i}\right)$ is about $\lambda_{g} / 2\left(\lambda_{g}\right.$ being the wavelength in the substrate located between the inner and outer conductors). The distance between two slots $\left(Z_{i}\right)$ is set to be around $\lambda_{g} / 4$ along the slant height of the outer conductor. Consequently, they are excited with a $90^{\circ}$ phase difference and, hence, the two magnetic current elements create the $\mathrm{CP}$ property [7]. It is noted that $\alpha_{i}$ can be used as a fine tuning parameter to improve the AR performance. The simulated electric field on the outer conductor at $5.7 \mathrm{GHz}$ is illustrated at different time instants in Fig. 2. It is evident that the electric field rotates in a clockwise direction with almost the same amplitude and thus radiates a left hand $\mathrm{CP}$ (LHCP) wave.

The two sets of slot pairs are arranged along the $\mathrm{Z}$ axis. Because they lie on a cone, the radii of and the number of slot pairs in both sets are not equal. The optimized design has 6 slot pairs in the upper set and 12 in the lower set. This choice yields more symmetry and more uniform gain. The length of the slots of the upper set $\left(L_{1}\right)$ is a little different from that of the lower set $\left(L_{2}\right)$. This choice yields some bandwidth expansion and aids the impedance matching. The distance, $H_{2}$, between the two sets of slot pairs along the slant height of the outer conductor is set to be about $\lambda_{g}$. This choice causes them to be excited in phase and, consequently, the radiated beam direction to be perpendicular to the outer conductor. For the sake of compactness, the slant height distance, $H_{l}$, between the top surface and the upper sets of the slot pairs and the slant height distance, $H_{3}$, between the bottom surface and the lower sets of slot pairs should be as short as possible. Therefore, $H_{l}$ was selected just long enough to support the upper set of slot pairs. Any reflections that might occur from the bottom surface can be reduced by adjusting the width of the slot pairs, $W_{l}$ and $W_{2}$, and the distance $H_{3}$ to ensure that as little as possible energy be left after the power flows past the lower set of slot pairs.

The antenna is excited by a single coaxial cable. As indicated in Fig.1(b), its inner conductor is connected directly to the top surface of the truncated cone structure. A smooth transition at the connection point is needed because it is where the power gets launched onto the outer conductor. Once past this connection point, the power flows along the outer conductor down to the lower part of the antenna, exciting the slot radiators. Fig. 3 shows this power flow when the antenna is operating at 5.7 $\mathrm{GHz}$. One immediately sees that most of the power is radiated from both arrays of resonant slots leaving only a minimal amount of energy at the bottom of the cone structure. As a result, the reflections from the bottom surface are tiny yielding a good traveling wave property and good AR results.

The antenna was designed through a series of simulations using CST Microwave Studio software. The parameters of the optimized configuration are summarized in Table I.

\section{PARAMETER ANALYSIS}

The transition area at the top surface is vital to the return loss performance and for fabrication convenience, we take $S_{l}$ approximately equal to the radii of $50 \Omega$ through coaxial line's 


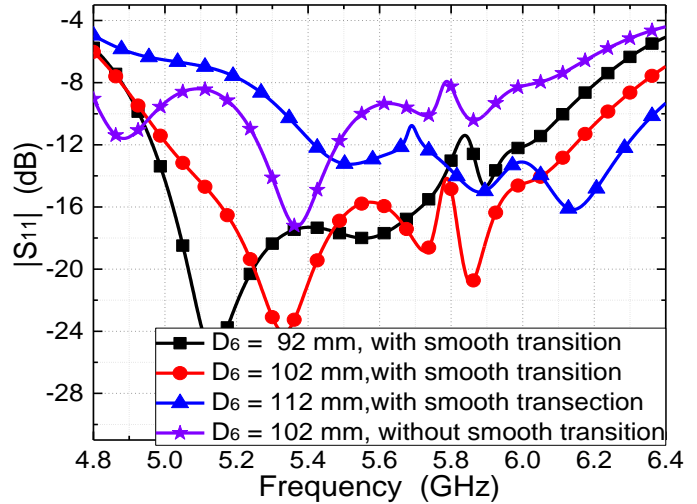

Fig. 4. Comparison of the simulated $\left|S_{11}\right|$ values for the $\mathrm{CP}$ conical beam antenna with and without the smooth transition element and the effect of bottom diameter of the inner conductor $D_{6}$ on the simulated $\left|S_{11}\right|$ values.

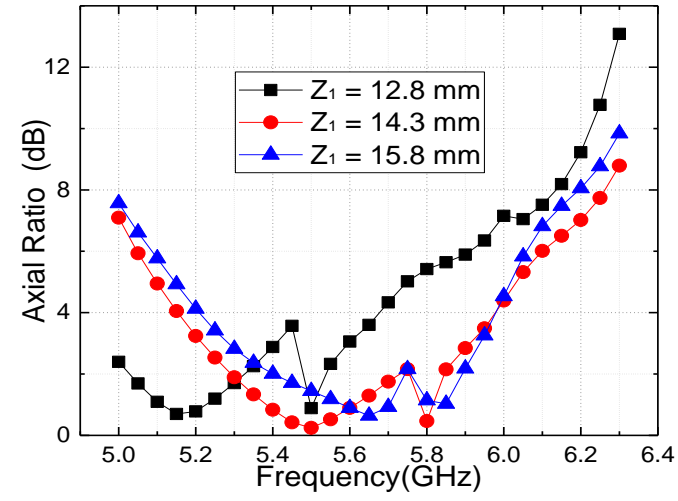

Fig. 5. Effect of the length between two slots of the upper set of slot pairs, $Z_{1}$, on AR results.

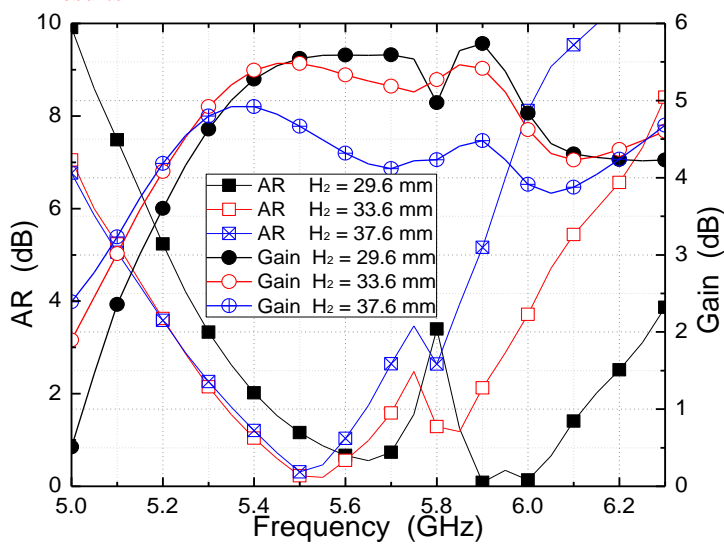

Fig. 6. Effect of the distance, $H_{2}$, between the two sets of slot pairs along the slant height on the simulated $\mathrm{AR}$ and realized gain values.

outer-conductor. The through coaxial line excites the slot arrays from the top to the bottom without increasing the height of the antenna. If one were to excite the slot arrays from the bottom to the top, a tapered coaxial line would have to be added to connect the small SMA port to the much larger bottom plane of the antenna. Apart from the transition area, the bottom diameter of the inner conductor $D_{6}$ was also a vital parameter for impedance matching. Notice that the inner and outer conductors (and in-between substrate) form a gradient coaxial line. This aspect is important for realizing equal radiated power from both sets of slot pairs and for the impedance matching.

In order to reduce reflections from the top surface, a small metal, inverted truncated cone is introduced between the inner conductor of the coaxial line and the bottom of the top surface.
The $\left|S_{11}\right|$ values with and without this transition element are plotted in Fig. 4. It is clear that the $\left|S_{11}\right|$ performance is significantly improved when this transition element is present. It was determined that the impedance matching will deteriorate if $D_{6}$ deviates from $102 \mathrm{~mm}$. The bottom diameter of the inner conductor controls the gradient impedance characteristic. If $D_{6}$ is less than $102 \mathrm{~mm}$, the $\left|S_{11}\right|$ values in the higher band will deteriorate, and the upper set of slot pairs will not be properly matched. If $D_{6}$ is larger than $102 \mathrm{~mm}$, the $\left|S_{11}\right|$ values in lower band will deteriorate, and the lower set of slot pairs will not be properly matched. Moreover, the distance gap between the centers of the two slots in the quasi-perpendicular pair, $Z_{l}$ and $Z_{2}$, is critical to the AR performance. If the gap is set correctly, the two slots in a pair will be excited with the desired $90^{\circ}$ phase difference, yielding the $\mathrm{CP}$ radiation. Any offset from the optimized value will cause poor AR performance, which is shown in Fig. 5.

If the two sets of slots are excited with the same phase and produce equal amounts of radiated power, the maximum gain in the direction perpendicular to the radiator's surface will be attained. The distance parameter $H_{2}$ affects this phase difference the most. Moreover, as Fig. 6 shows, it also impacts the AR and realized gain values. This effect occurs because it controls the current distributions. The choice, $H_{2}=$ $33.6 \mathrm{~mm}$, was a compromise between these two issues.

\section{RESULTS AND DISCUSSION}

A prototype of the optimized $\mathrm{CP}$ conical beam antenna was fabricated and tested. Fig. 7 compares the measured and simulated $\left|S_{11}\right|$ values. A photo of the antenna under test (UAT) is also included. The measured $-10-\mathrm{dB}$ impedance bandwidth (i.e., $\left|S_{11}\right|<-10 \mathrm{~dB}$ ) is $720 \mathrm{MHz}$, ranging from 5.05 to $5.77 \mathrm{GHz}$. This outcome is somewhat deteriorated from the simulated results. The discrepancy is attributed to fabrication inaccuracies and an imprecise substrate permittivity value.

Fig. 8 demonstrates that the measured AR bandwidth is 0.5 $\mathrm{GHz}$, from 5.5 to $6.0 \mathrm{GHz}$. Again, this is less than the simulated value. The difference occurs at the upper end of the AR bandwidth. Because the AR value is sensitive to the position of the slot pairs, the discrepancy is mainly due to fabrication inaccuracies. Within the AR bandwidth, the measured LHCP realized gain values show the same trend as the simulated ones and are found to be between 4.84 and $5.8 \mathrm{dBic}$.

The measured LHCP normalized radiation pattern, the simulated, normalized LHCP and RHCP radiation patterns in the E-plane and $45^{\circ}$ elevation angle plane at $5.6 \mathrm{GHz}, 5.8 \mathrm{GHz}$, $6.0 \mathrm{GHz}$ are shown in Fig. 9. From the simulated results, it is clear that the cross-polarization levels are at least $16 \mathrm{~dB}$ lower than the co-polarization values and that the maximum gain direction remains almost unchanged and, as designed, points to about $45^{\circ}$ from boresight within the operational band. The measured maximum realized gain direction is also around $45^{\circ}$. However, notice that the gain values are higher than the simulated ones in the direction of $15^{\circ}$ from boresight. Again, simulations have determined that this discrepancy is mainly due to the fabrication inaccuracies and test errors. Table II compares the performance of the different types of $\mathrm{CP}$ conical beam antennas. It can be noted that the main advantage of the proposed antenna is that it can be redesigned to radiate any 


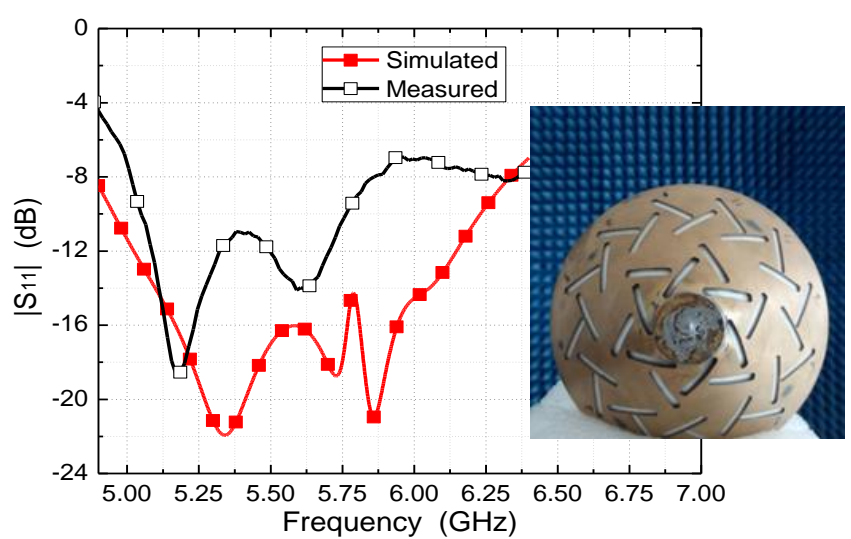

Fig. 7. Comparison of the simulated and measured $\left|S_{11}\right|$ values of the $C P$ conical beam antenna.

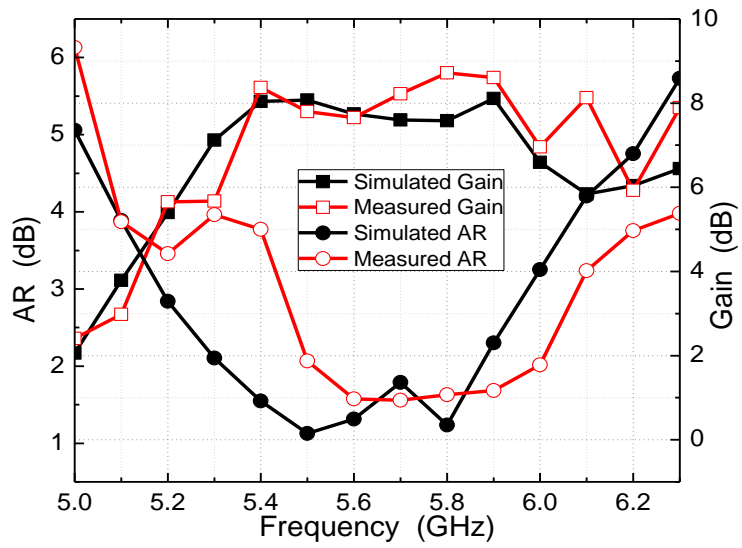

Fig. 8. Comparison of the simulated and measured realized gain and AR values of the $\mathrm{CP}$ conical beam antenna in the direction perpendicular to the outer conductor.

TABLE II

PERFORMANCE COMPARISON OF CP CONICAL BEAM ANTENNAS

\begin{tabular}{|c|c|c|c|c|c|}
\hline Literature & $\begin{array}{c}\text { AR } \\
\text { bandwi } \\
\text {-dth }\end{array}$ & Profile & $\begin{array}{c}\text { Max. } \\
\text { Gain } \\
(\mathrm{dB})\end{array}$ & $\begin{array}{c}\text { Network } \\
\text { Required }\end{array}$ & $\begin{array}{c}\text { Range of the } \\
\text { beam pointing } \\
\text { angle }\end{array}$ \\
\hline$[1]$ & $28.3 \%$ & $0.084 \lambda_{0}$ & 5.5 & Yes & $\sim 33^{\circ}$ \\
\hline$[2]$ & $2.66 \%$ & $0.069 \lambda_{0}$ & 6.5 & Yes & $\sim 46^{\circ}$ \\
\hline$[4]$ & $5 \%$ & $\lambda_{0}$ & 7.5 & Yes & $\sim 28^{\circ}$ \\
\hline$[5]$ & $14.4 \%$ & $0.06 \lambda_{0}$ & 4.9 & No & $\sim 32^{\circ}$ \\
\hline$[7]$ & $14.5 \%$ & $4.3 \lambda_{0}$ & 7 & No & $\sim 90^{\circ}$ \\
\hline$[3]$ & $4 \%$ & $0.19 \lambda_{0}$ & $3.8 \sim 6.9$ & Yes & $35^{\circ} \sim 74^{\circ}$ \\
\hline$[6]$ & - & $0.119 \lambda_{0}$ & $3 \sim 9$ & No & $10^{\circ} \sim 60^{\circ}$ \\
\hline Prototype & $8.7 \%$ & $\lambda_{0}\left(45^{\circ}\right)$ & 5.8 & No & $0^{\circ} \sim 90^{\circ}$ \\
\hline
\end{tabular}

angle CP beam with high gain along the boresight direction to meet the actual requirements.

\section{CONCLUSION}

In this paper, a circular truncated cone slot antenna that radiates a LHCP beam at $45^{\circ}$ with respect to boresight was studied. The measured prototype demonstrated a $720 \mathrm{MHz}$ impedance bandwidth, covering 5.05 to $5.77 \mathrm{GHz}$ and a 500 MHz 3-dB AR bandwidth, from 5.5 to $6.0 \mathrm{GHz}$. The measured realized gain over this AR bandwidth ranged from 4.84 to 5.8 $\mathrm{dBi}$. It is noted that by making one slot in each pair be inclined $\alpha_{i}$ degrees anticlockwise while making the other $\alpha_{i}$ degrees clockwise, RHCP performance can be realized, Moreover, by adding more sets of slot pairs and adjusting the width of slots, higher realized gain can be realized. Finally, the direction of the
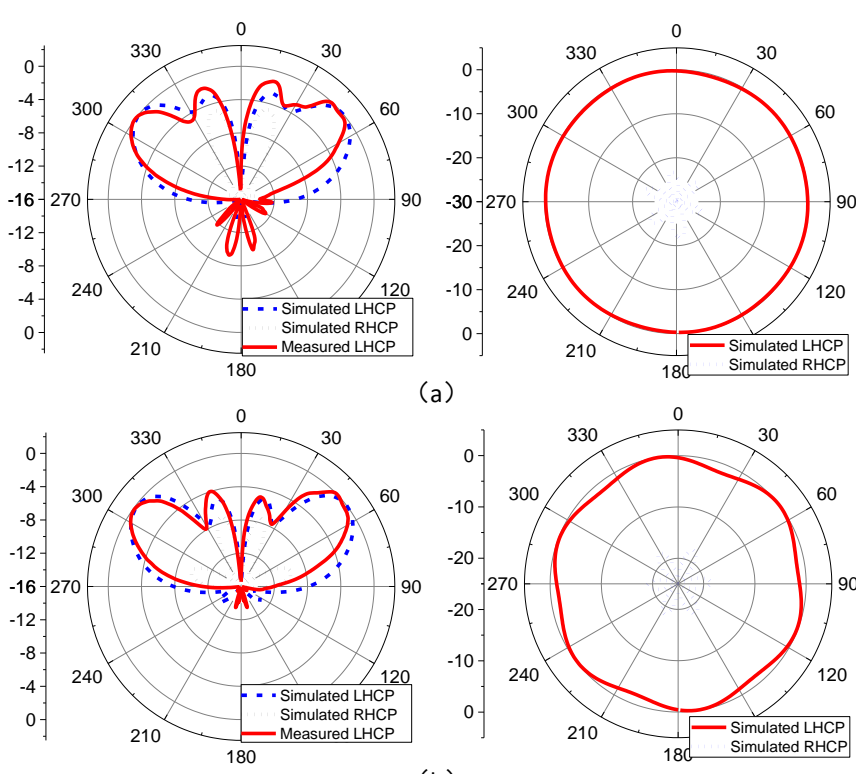

(a)
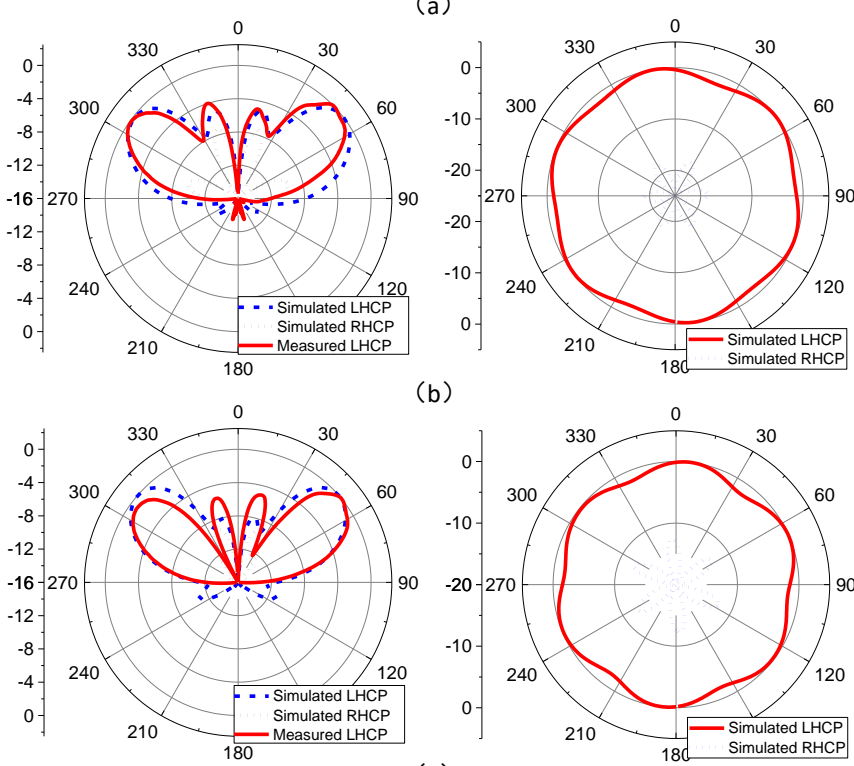

(b)

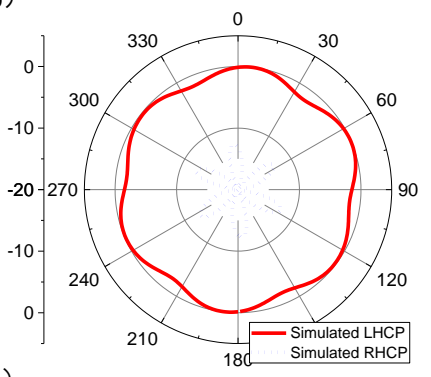

(c)

Fig. 9. Comparisons of the normalized, measured LHCP and simulated LHCP and RHCP realized gain patterns in the E-plane and $45^{\circ}$ elevation angle plane at (a) $5.6 \mathrm{GHz}$, (b) $5.8 \mathrm{GHz}$, (c) $6.0 \mathrm{GHz}$.

beam was demonstrated to be perpendicular to the outer conductor. Consequently, it is easy to change the beam direction simply by changing the slant angle of the outer conductor to meet the actual requirements.

\section{REFERENCES}

[1] K.L.Lau, K.M.Luk, "A wideband circularly polarized conical-beam patch antenna," IEEE Trans. Antennas Propag., vol. 54, pp. 1591-1594, 2006.

[2] X.Bai, X.Liang, M.Li, et al. "Dual-circularly polarized conical-beam microstrip antenna," IEEE Antennas Wireless Propag. Lett., vol. 14, pp. 482-485, 2015

[3] J.Huang, "Circularly polarized conical patterns from circular microstrip antennas," IEEE Trans. Antennas Propag., vol. 32, pp. 991-994, 1984.

[4] S.S.Qi, Wu.W, Fang.D.G, "Singly-fed circularly polarized circular aperture antenna with conical beam," IEEE Trans. Antennas Propag., vol. 61, pp. 3345-3349, 2013.

[5] W.Lin, H.Wong, "Circularly polarized conical-beam antenna with wide bandwidth and low profile," IEEE Trans. Antennas Propag., vol. 62, pp. 5974-5982, 2014,.

[6] J.Takada, A.Tanisho, K.Ito, et al. "Circularly polarised conical beam radial line slot antenna,” Electronics Lett., vol. 30, pp. 1729-1730, 1994.

[7] B.Zhou, J.Geng, X.Bai, et al. "An omnidirectional circularly polarized slot array antenna with high gain in a wide bandwidth," IEEE Antennas Wireless Propag. Lett., vol.14, pp. 666-669, 2015. 